\title{
Conhecimentos e usos da fauna por caçadores no semiárido brasileiro: um estudo de caso no estado da Paraíba, Nordeste do Brasil
}

\author{
José Aécio Alves Barbosa * \\ José Otávio Aguiar \\ Programa de Pós-Graduação em Recursos Naturais, Universidade Federal de Campina Grande \\ Avenida Aprígio Veloso, 882, Bodocongó, CEP 58429-140, Campina Grande - PB, Brasil \\ * Autor para correspondência \\ barbosa.joseaecioalves@gmail.com
}

Submetido em 13/07/2014

Aceito para publicação em 04/02/2015

\section{Resumo}

Esse estudo foi realizado em uma comunidade tradicional no semiárido paraibano e registrou as principais espécies animais caçadas, avaliando as implicações conservacionistas desta prática. Um total de 24 entrevistados citou 78 espécies de vertebrados tetrápodes silvestres caçados na região. Esses animais podem ser organizados, de acordo com o uso, em dez categorias diferentes: zooterapia, etnoveterinária, uso cosmético, uso/interação místico-religiosa, criação/domesticação, comércio, uso/interação ornamental, alimentação, lazer/recreação, caça de controle. Com o presente trabalho percebeu-se que a carência de estudos em relação às formas de aproveitamento dos recursos faunísticos constitui uma barreira que dificulta o levantamento das prioridades de conservação e manejo, por impossibilitar um inventário seguro das espécies animais que sofrem maior pressão de uso. Entretanto, mesmo com essa barreira, constatamos seguramente que a interação de aproveitamento e emprego existente entre homem e fauna constitui uma das mais significativas formas de aplicação dos recursos naturais nas áreas estudadas.

Palavras-chave: Caatinga; Caça; Conservação; Usos da fauna

\section{Abstract}

Knowledge and use of wildlife by hunters in the Brazilian semiarid region: a case study in Paraíba State. The present study was undertaken in a traditional community in the semiarid region of Paraíba State, Northeastern Brazil, with the purpose of registering the main animal species hunted and evaluating the implications of these practices in terms of the conservation of local fauna. A total of 78 species of wild tetrapod vertebrates were cited by 24 interviewees as being hunted in the region. These animals were separated into four different animals groups (mammals, birds, reptiles and amphibians) with ten distinct purposes: zootherapy, folk veterinary medicine, cosmetics, mystical-religious purposes, creation/domestication, commercial exploration, ornamental use, as food, as entertainment, and for animal control. This work highlights the lack of studies regarding some forms of exploitation of wildlife resources as a barrier that hinders the assessment of priorities for conservation and management, by precluding a stable inventory of animal species under the greatest pressure of use. However, even with these barriers we can clearly see that exploitation and utilization of wildlife by man represents one of the most significant uses of natural resources in the surveyed areas.

Key words: Caatinga biome; Conservation; Hunting; Wildlife uses 


\section{Introdução}

As relações que historicamente se estabelecem entre os humanos e os animais representam uma das mais constantes formas de interação do homem com a biodiversidade (ALVES et al., 2009). Essa variedade de interações que as culturas humanas mantêm com a fauna é comumente abordada pela perspectiva da etnozoologia, um saber subdiferenciado que investiga os conhecimentos, significados e usos dos animais nas sociedades humanas (OVERAL, 1990; COSTA-NETO, 1999; MARQUES, 2002).

Mesmo sendo essa uma atividade considerada ilegal no Brasil (Lei no 5.197/1967), várias espécies continuam sendo abatidas em no país (TRINCA; FERRARI, 2006; GUADAGNIN et al., 2007; HANAZAKI et al., 2009), inclusive em áreas semiáridas (ALVES et al., 2009; BARBOSA et al., 2011), o que conjuntamente à degradação dos habitats potencializa o status de ameaça ambiental nessas regiões de menor incidência de chuvas (MMA, 2002; 2003; 2009; IBAMA, 2003; LEAL et al. 2005). Mesmo diante desse cenário, poucos estudos etnozoológicos têm examinado as atividades de caça no semiárido (ALVES et al., 2007) e pouca atenção tem sido direcionada ao tema.

Essa prevalência de atividades ilegais de caça no semiárido brasileiro gera conflitos ambientais e é, em grande parte, motivada por questões socioculturais e econômicas (BARBOSA et al., 2012), tendo em vista a importância nutricional das carnes provenientes de animais selvagens (ROUBIK, 1995) como fonte de proteína para as populações humanas (SMITH, 1976; AYRES; AYRES, 1979; MARTINS, 1993; CALOURO, 1995; EMÍDIO-SILVA, 1998). Além disso, diversos animais são amplamente utilizados nessas áreas para fins medicinais, mágico-religiosos, cosméticos, ornamentais e de estimação (ALVES et al., 2007; 2009; 2011; BARBOSA et al., 2010). No demais, outros ainda são caçados por seu eventual potencial para atacar seres humanos ou criações domésticos (BARBOSA et al., 2011; MENDONÇA et al., 2012).

Diante dessas diferentes formas de aproveitamento dos recursos faunísticos, que por vezes podem constituir ameaça à biodiversidade, faz-se necessário conhecer as principais motivações da atividade cinegética e as espécies mais exploradas, no intúito de possibilitar a proposta adequada de medidas conservacionistas cabíveis sem, contudo, ameaçar identidade cultural e a estabilidade socioeconômica local. Nesse sentido o presente estudo, realizado em uma área da região semiárida do estado da Paraíba, objetivou melhor descrever as práticas de caça no semiárido brasileiro, registrando as principais espécies caçadas e as motivações dessa atividade, para assim avaliar as implicações dessa prática em termos de conservação da fauna local.

\section{Material e Métodos}

\section{Da área de estudo}

O presente trabalho foi desenvolvido no município de Fagundes (latitude $7^{\circ} 20^{\prime} 45.56$ "S; longitude $35^{\circ} 47^{\prime} 51.13$ ” W), localizado no semiárido do estado da Paraíba (Mesorregião Agreste), Brasil (Figura 1). Esse município foi escolhido por ser de fácil acesso e apresentar características predominantemente rurais, com comunidades tradicionais compostas por sertanejos típicos.

O município de Fagundes está inserido predominantemente na unidade geoambiental do Planalto da Borborema, em um trecho que se aproxima da Depressão do Rio Paraíba, e representa a paisagem típica do semiárido nordestino. Seu relevo é predominantemente suave-ondulado, cortada por vales estreitos, com vertentes dissecadas. A vegetação é basicamente composta por Caatinga Hiperxerófila com trechos de Floresta Caducifólia e pequenas áreas transicionais de Mata Atlântica. O clima é do tipo Tropical Semiárido, com chuvas de outono-inverno e precipitação média anual de 431,8 mm (CPRM, 2005; IBGE, 2010).

Fagundes possui uma área de $162 \mathrm{~km}^{2}$. A sede do município tem uma altitude aproximada de $505 \mathrm{~m}$, distando $104 \mathrm{~km}$ da capital. O município foi criado em 1961 e possui uma população total de 11.409 habitantes, sendo 5.942 na zona rural. Seu índice de Desenvolvimento Humano (IDH) é de 0,559 , segundo o Atlas de Desenvolvimento Humano-PNUD - 2000 (CPRM, 2005; IBGE, 2010). 
FIGURA 1: Localização da área estudada - município de Fagundes, semiárido do estado da Paraíba, Região Nordeste do Brasil.

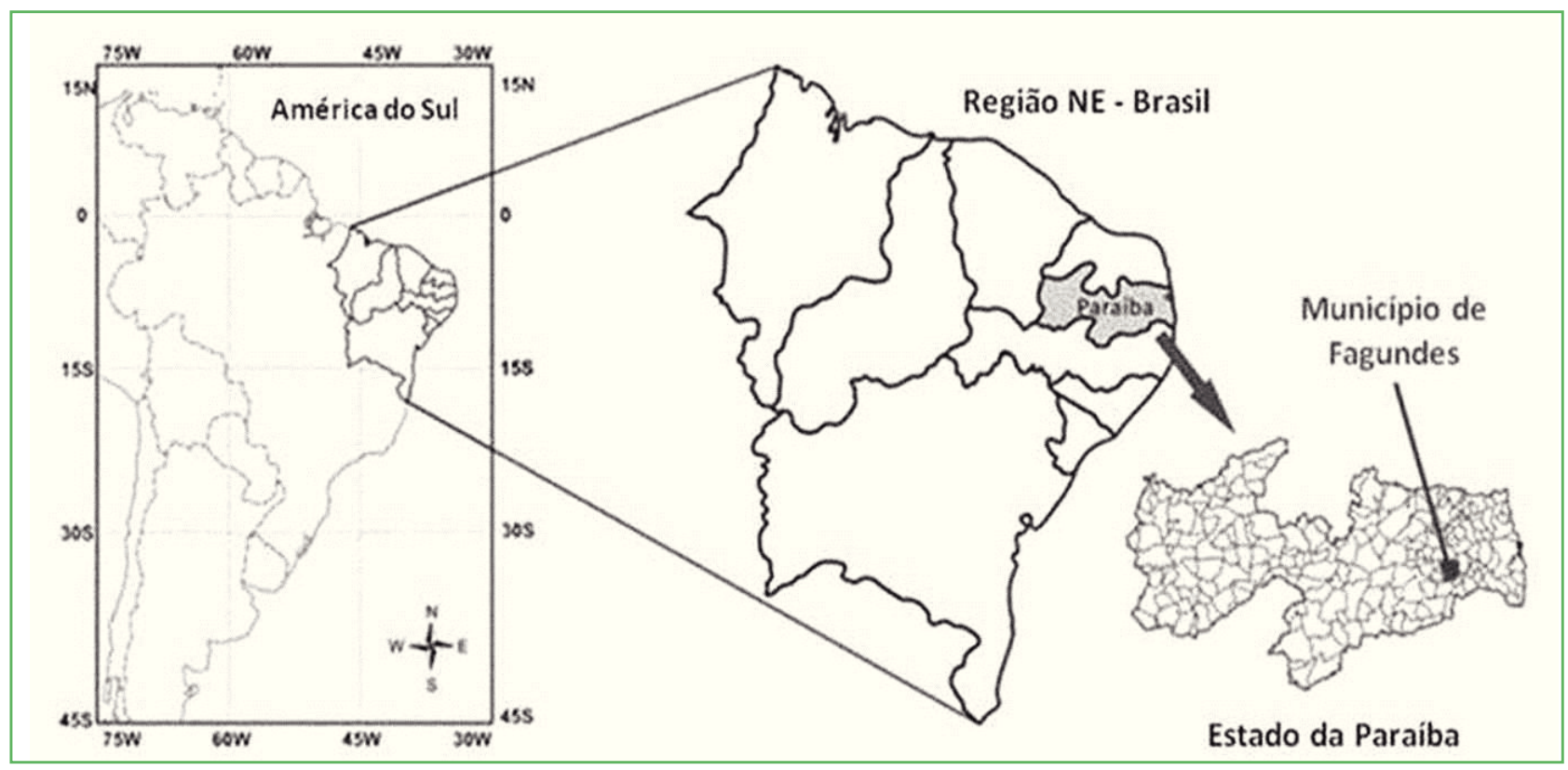

Há cerca de 130 anos atrás, as florestas próximas às comunidades pesquisadas eram muito abundantes quanto aos recursos de caça, o que, consequentemente, moveu parte da população a deslocar-se até lá. Essas pessoas começaram a povoar a região e, desde então, ainda desenvolvem atividades de caça em torno da área. Entretanto, a agropecuária de subsistência é a prática mais comum, com o cultivo de feijão, milho, mandioca e batata doce, e a criação de bovinos, caprinos, ovinos, suínos, galináceos e outros animais (ALVES et al., 2011).

\section{Coleta e análise dos dados}

A pesquisa de campo compreendida neste trabalho foi realizada entre dezembro de 2009 e dezembro de 2012. A primeira etapa da pesquisa envolveu entrevistas com os caçadores da localidade. Após os primeiros contatos, informações detalhadas sobre as práticas de caça foram obtidas através da aplicação de formulários semiestruturados (HUNTINGTON, 2000; ALBUQUERQUE; LUCENA, 2004), complementados com entrevistas livres e individuais (MARQUES, 1991; MELLO, 1995; CHIZOTTI, 2000; ALBUQUERQUE; LUCENA, 2004). O formulário semiestruturado continha perguntas acerca da biologia e ecologia dos animais que eram caçados, como eles eram capturados e quais as motivações principais da caça.

Os nomes comuns das espécies foram listados conforme mencionados pelos entrevistados e os animais foram posteriormente identificados por: 1) análise dos espécimes doados pelos entrevistados, 2) análise de fotografias dos animais feitas durante as entrevistas; 3) correlação entre os nomes comuns, com o auxílio de taxonomistas familiarizados com a fauna na área de estudo (ALVES; ROSA, 2006). A pesquisa foi aprovada pelo comitê de ética em pesquisa da UFCG (Protocolo CAAE: 0013.0.167.000-05).

Para cada espécie de animal citada foi calculado seu respectivo Valor de Uso (VU), seguindo Rossato et al. (1999) e adaptado por Lucena et al. (2012). O valor de uso (atual e potencial) foi calculado através das seguintes fórmulas:

$$
\left(\boldsymbol{V} \boldsymbol{U}_{A}=\boldsymbol{\Sigma} \boldsymbol{U}_{A} / \boldsymbol{n}\right) \mathrm{e} / \mathrm{ou}\left(\boldsymbol{V} \boldsymbol{U}_{\boldsymbol{P}}=\boldsymbol{\Sigma} \boldsymbol{U}_{\boldsymbol{P}} / \boldsymbol{n}\right)
$$

Onde: $\mathrm{VU}_{\mathrm{A}}=$ valor atual de uso da espécie (espécies conhecidas e efetivamente utilizadas); $\mathrm{VU}_{\mathrm{P}}=$ valor potencial de uso da espécie (espécies de uso conhecido, porém não utilizadas atualmente); $\mathrm{U}_{\mathrm{A}}=$ número de citações de uso atual por espécie; $\mathrm{U}_{\mathrm{P}}=$ número de 
citações de uso potencial por espécie; $\mathrm{n}=$ número de informantes.

Essa diferenciação nos valores de uso foi importante no sentido de minimizar algumas limitações de cálculo, incluindo o fato de o índice clássico de Rossato et al. (1999) não fazer distinção entre o uso corrente e o uso potencial, o que poderia superestimar ou subestimar a análise.

O levantamento e apresentação desses dados podem ser úteis aos órgãos competentes no eventual processo de ordenamento e fiscalização das atividades de exploração da fauna na área estudada, e possibilita o envolvimento efetivo dos habitantes locais nas discussões sobre a obtenção desses recursos, de forma a contribuir com a conservação das espécies e manutenção da identidade cultural na região.

\section{Resultados e Discussão}

Vinte e quatro caçadores foram entrevistados, com idades variando entre 18 e 56 anos. Os entrevistados citaram um total de 78 espécies da fauna local que são abatidas e/ou utilizadas na região: Mamíferos (11), Aves (50), Répteis (14) e Anfíbios (3).

Dez diferentes formas de interação e uso da fauna local foram registradas no presente trabalho: Zooterapia (o uso de animais e seus derivados em tratamentos alternativos e saúde); Etnoveterinária (a utilização direta dos animais ou seus derivados em tratamentos veterinários alternativos); Uso Cosmético (a aplicação de subprodutos derivados da fauna em tratamentos estéticos); Uso/Interação Mistico-Religiosa (a inserção de animais em rituais mágico/religiosos, e a crença no potencial místico de certas espécies); Criação/Domesticação (manutenção em cativeiro de espécimes da fauna silvestre local, seja por motivação afetiva ou utilitária); Comércio (atividade financeira de aquisição ou venda de animais ou subprodutos derivados); Uso/Interação Ornamental (utilização de animais vivos ou seus subprodutos como artefatos de adorno pessoal ou de ambientes); Alimentação (aproveitamento nutricional das carnes e derivados animais); Lazer / Recreação (atividades de interação com a fauna motivadas por entretenimento e diversão); Caça de Controle (abate de espécimes considerados potencialmente perigosos para a população local e suas criações domésticas). Das modalidades registradas, as que mais se destacaram foram o uso alimentar $(24,37 \%$ das citações), o comércio de animais e subprodutos (16,21\%) e a zooterapia $(15,23 \%)$.

As Figuras 2 e 3 demonstram, respectivamente, o percentual de citações para cada modalidade de uso/ interação e a distribuição de cada grupo de vertebrados terrestres dentro dessas modalidades. Os Valores de Uso Atuais $\left(\mathrm{VU}_{\mathrm{A}}\right)$ das espécies citadas variaram de zero a 1,75; já os Valores de Uso Potenciais $\left(\mathrm{VU}_{\mathrm{p}}\right)$ variaram de zero a 1,17. A grande maioria das espécies apresentou valores de uso baixos (menores que 0,3). Ainda assim, 24 espécies tiveram VU acima de 0,5 , refletindo a diversidade de uso desses animais, com destaque no Valor de Uso Atual para o Teju - Tupinambis merianae $\left(\mathrm{VU}_{\mathrm{A}}=1,75\right)$; Peba - Euphractus sexcinctus $\left(\mathrm{VU}_{\mathrm{A}}=1,63\right)$, Tatu Dasypus novemcinctus $\left(\mathrm{VU}_{\mathrm{A}}=1,63\right)$; Ribaçã - Zenaida auriculata $\left(\mathrm{VU}_{\mathrm{A}}=1,33\right)$; Rolinha - Columbina sp. $\left(\mathrm{VU}_{\mathrm{A}}\right.$ $=1,33)$; Preá - Cavia aperea $(\mathrm{VU}=1,08)$ e Cascavel - Caudisona durisa (VU=1,04), e no Valor de Uso Potencial para Raposa - Cerdocyon thous $\left(\mathrm{VU}_{\mathrm{P}}=1,17\right)$; Gato-maracajá - Leopardus wiedii $\left(\mathrm{VU}_{\mathrm{P}}=0,58\right)$ e Cancão - Cyanocorax cyanopogon $\left(\mathrm{VU}_{\mathrm{P}}=0,54\right)$. $\mathrm{O}$ anexo I expõe detalhadamente os animais citados, suas utilidades locais e os respectivos valores de uso atuais e potenciais.

Das espécies registradas e com Valor de Uso Atual acima de 1, mais de 70\% são mamíferos ou aves. Esse elevado $\mathrm{VU}_{\mathrm{A}}$ é um indício de que essas espécies, apesar de ainda presentes na região, podem estar sendo sobre-exploradas localmente. A importância atual de mamíferos e aves para a população local é, sobretudo, motivada pela utilização alimentar desses animais. Segundo os entrevistados, a preferência pela exploração desses grupos faunísticos se dá, especialmente, por dois fatores: a relativa abundância desses animais em comparação com outros grupos de vertebrados e seu porte médio, que implica em um maior retorno de produtos e subprodutos a cada caçada. Trinca e Ferrari (2006) observaram que os caçadores da Amazônia do estado do Mato Grosso também relacionavam os animais abatidos à abundância local e ao porte.

É evidente que espécies que provêm maior quantidade de produtos e subprodutos para o homem 
FIGURA 2: Percentual de citações para cada modalidade de uso/ interação com a fauna cinegética na localidade estudada.

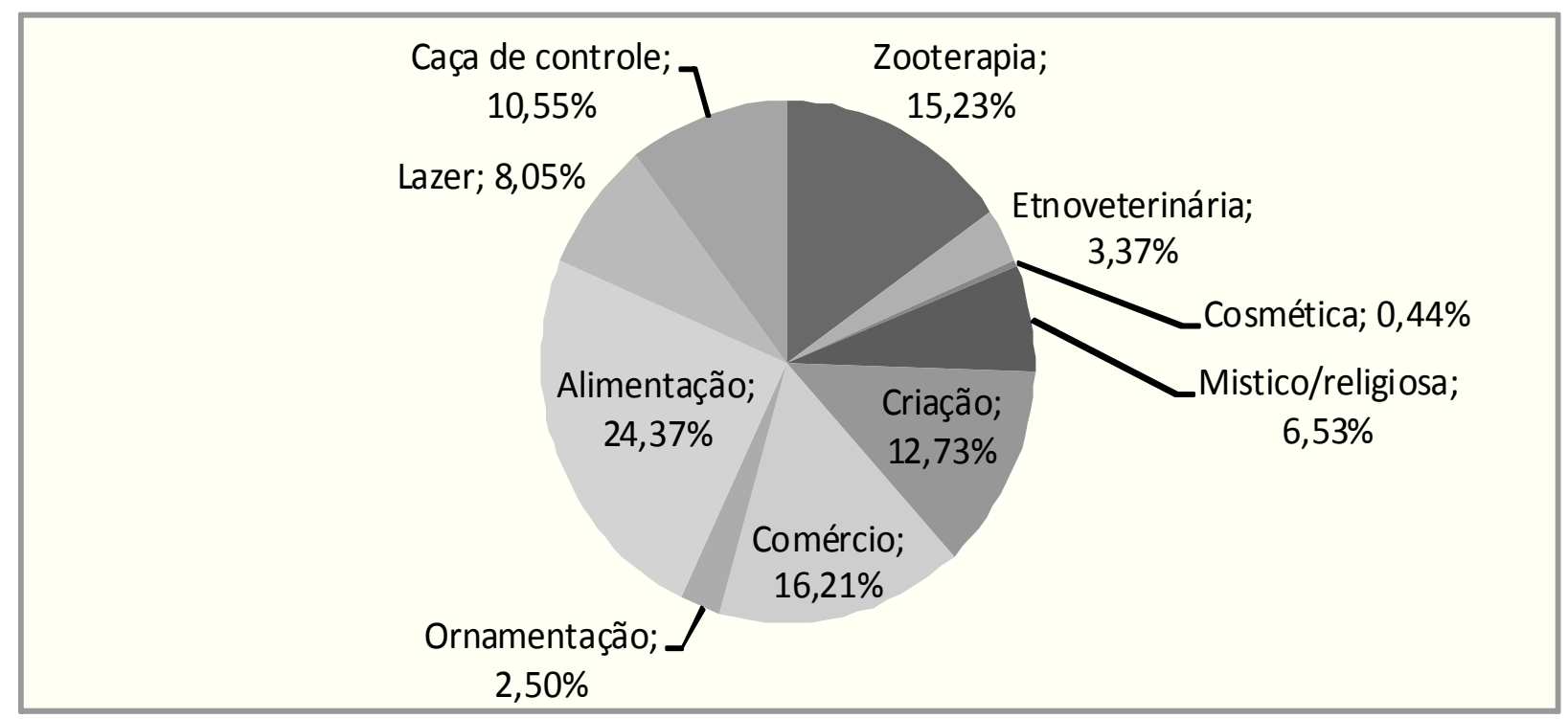

FIGURA 3: Distribuição dos grupos zoológicos por modalidade de uso da fauna cinegética local. Legenda: Zooterapia (A); Etnoveterinária (B); Uso cosmético (C); Uso/Interação místico-religiosa (D); Criação/Domesticação (E), Comércio (F); Uso/Interação ornamental (G); Alimentação (H); Lazer/Recreação (I); Caça de controle (J).

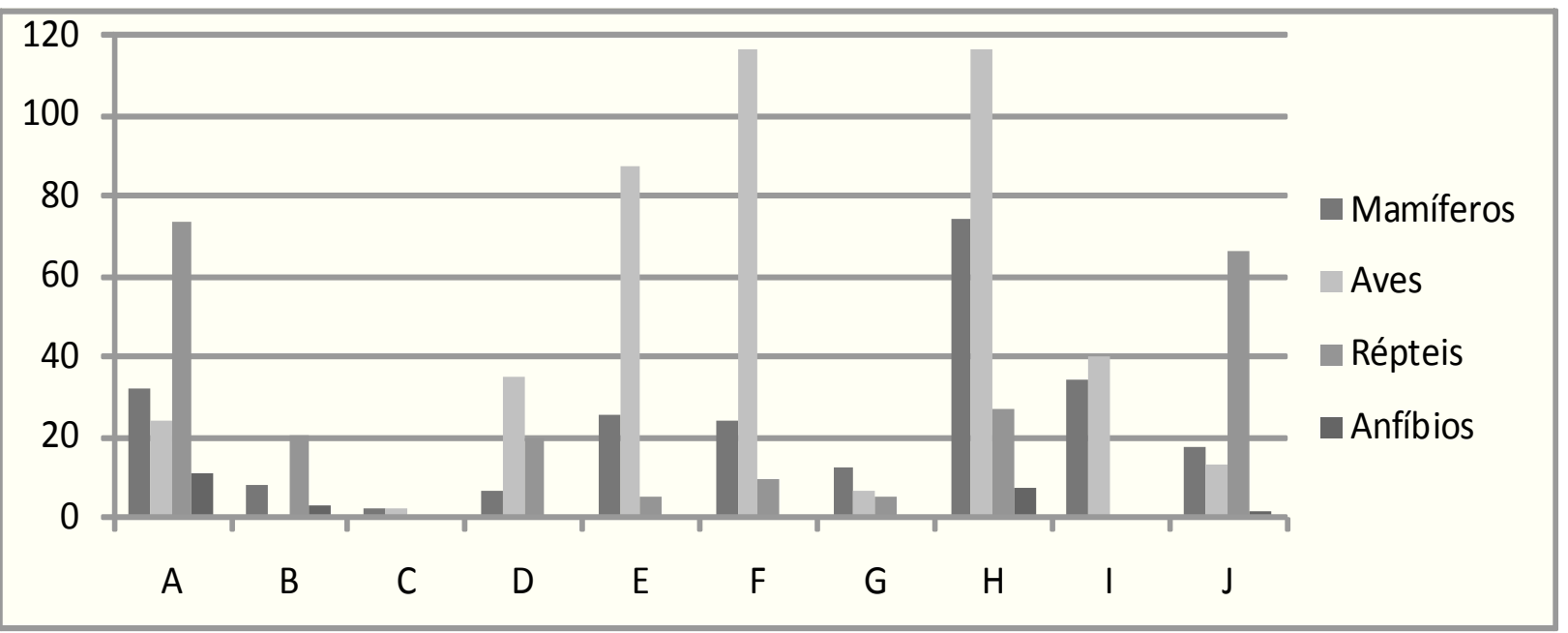


usualmente são as mais exploradas (BODMER; PEZO, 2001; ZAPATA, 2001; MILNER-GULLAND et al., 2003). Nesse sentido, parece ser recorrente e constante que na região os mamíferos e as aves venham sendo os grupos de vertebrados silvestres com maior histórico de aproveitamento (ALVES et al., 2009). Em estudo realizado em comunidades indígenas "Tupinambás" no município de Ilhéus, Bahia, Pereira e Schiavetti (2010) registraram um maior índice de aproveitamento de mamíferos, seguido de aves. Martínez (2006), em estudos acerca da utilização da fauna silvestre pela comunidade do Petenes, adjacente a uma reserva biológica no Campeche, sudeste do México, também registrou como principais alvos de caça os mamíferos e as aves. Assim exposto, a reentrância dessas práticas cinegéticas na localidade aqui estudada precisa ser mais bem avaliada, no intuito de averiguar se existe sobre-exploração da fauna e implicações negativas para conservação desses recursos na área.

Assim como um elevado $\mathrm{VU}_{\mathrm{A}}$ é preocupante no sentido da sobre-exploração local, um Valor de Uso Potencial quando elevado pode refletir a ausência desses animais na região, fato observado para a Raposa - Cerdocyon thous $\left(\mathrm{VU}_{\mathrm{P}}=1,17\right)$ e o Gato-maracajá Leopardus wiedii $\left(\mathrm{VU}_{\mathrm{p}}=0,58\right)$, e agravado pelo relato, por parte dos caçadores entrevistados, da escassez desses animais nos habitats locais. Tanto a utilização efetiva atual quanto o relato da diminuição local de alguns mamíferos na região deixa clara a necessidade urgente de trabalhos de cunho conservacionista voltados para esses animais na área estudada.

Além da alimentação, mais um estímulo à captura de animais silvestres nas áreas pesquisadas é o valor comercial destes. O comércio ilegal de animais silvestres é um negócio que movimenta por ano entre 10 e 20 bilhões de dólares (VANNUCCI-NETO, 2000). O Brasil participa deste mercado com cerca de US\$ 1 bilhão ao ano, uma vez que sua enorme diversidade biológica, bem como as dificuldades sociais e os motivos culturais, fazem do país um dos principais fornecedores de animais silvestres do mundo (GIOVANINI, 2002).

Algumas espécies de mamíferos, aves e répteis são capturadas e criadas durante algum tempo para engorda e revenda de carne na localidade estudada, entretanto, a maior parte dos animais silvestres listados como criados e comercializados pelos entrevistados é de pássaros canoros, com pelo menos oito espécies de $\mathrm{VU}_{\mathrm{A}}$ acima de $(0,3)$ - Azulão - Passerina brissonii, Cancão Cyanocorax cyanopogon, Casaca-de-couro-Furnarius leucopus, Concriz - Icterus jamacaii, Corda-negra - Agelaius ruficapillus, Galo-de-campina - Paroaria dominicana, Golado - Sporophila albogularis e Sabiá - Turdus rufiventris, o que demostra que essas espécies são atualmente exploradas pelo comércio ilegal da fauna silvestre na região.

Dentre os animais vendidos, as aves, devido a sua beleza e vocalização (PEREIRA; BRITO, 2005), bem como a sua ampla disseminação geográfica e elevada variedade, são o grupo de animais mais procurados, inclusive na região pesquisada, como já havia sido evidenciado por Nobrega et al. (2011).

$\mathrm{Na}$ área de abrangência desta pesquisa, registrase uma preferência pela captura e comercialização de pássaros machos. Uma tendência similar foi observada por Rocha et al. (2006), que, em estudo acerca da comercialização de aves em feiras livres de Campina Grande, Paraíba (local onde a maioria dos pássaros canoros capturados na área aqui estudada é revendida), observaram métodos seletivos de captura de pássaros machos para revenda. Segundo Ribeiro e Silva (2007), uma captura acentuada de machos é um fato agravante para o desequilíbrio populacional das espécies de pássaros envolvidos, uma vez que cerca de 90\% das espécies de aves adotam um comportamento monogâmico durante seu período reprodutivo. Logo, alterações no equilíbrio reprodutivo das aves na região estudada pode afetar consideravelmente a biodiversidade local, fato que merece ser mais bem investigado.

Grande parte dos criadores e comerciantes de pássaros canoros da comunidade pesquisada apresentou um nível de reconhecimento e percepção bastante elevado no que diz respeito à vocalização das aves. Alguns ainda associaram esse fenômeno à reprodução. Um fato similar é apresentado por Cadima e Marçal Júnior (2004), que em um trabalho de investigação acerca do conhecimento etnoornitológico de habitantes do distrito rural de Miraporanga, Uberlândia, evidenciaram que essa característica repleta de significados presente no canto 
das aves foi claramente reconhecida por seus informantes. Almeida et al. (2006), em um estudo etnoornitológico realizado no distrito rural de Florestina, município de Araguari, região do Triângulo Mineiro, afirmam que os moradores da região mostraram conhecer diversos aspectos da biologia e da ecologia das aves, incluindo vocalização, reprodução, alimentação e características comportamentais. Esse elevado nível de percepção registrado aqui pode ser beneficamente empregado em estratégias de conservação da fauna local, uma vez que o conhecimento ecológico tradicional é uma ferramenta extremamente útil para o manejo sustentável dos recursos naturais (POSEY, 1983; ALBUQUERQUE: LUCENA, 2004; BARBOSA, 2011).

Além da utilização das carnes na alimentação e do comércio de animais vivos, muitos subprodutos que não apresentam utilidade alimentar ou comercial, como gorduras, ossos, dentes, penas, dentre outros, são aproveitados de diferentes maneiras na área em questão. $\mathrm{O}$ uso de sobras poderia ser justificado como uma tentativa de maximizar os recursos obtidos dos ecossistemas locais (MOURA; MARQUES, 2008). Uma das principais formas de utilização desses subprodutos na área onde esta pesquisa foi desenvolvida é a zooterapia. Em estudo acerca dos aspectos da zooterapia popular, desenvolvido na comunidade do Remanso, na Chapada Diamantina, Moura e Marques (2008) relatam que mais da metade dos zooterápicos listados em sua pesquisa derivam de subprodutos animais inúteis para outros fins.

Os animais medicinais citados pelos entrevistados podem ser aproveitados inteiramente ou em partes avulsas. As partes citadas são: ossos, couro, cauda, pelos, penas, dentes, cabeça, estômago, intestinos, fígado, "fel" (bílis), gordura, maracá (chocalho de cobra) e secreções do corpo. A maioria dessas matérias-primas tem sido registrada em outros trabalhos acerca da utilização humana dos recursos zooterápicos no Brasil (BEGOSSI; BRAGA, 1992; COSTA-NETO, 1999; MARQUES, 2001; ALVES; ROSA, 2006; 2007a; 2007b; ALVES et al., 2007; 2011; ALVES, 2009), o que indica que essa prática é largamente difundida no país. Isto ratifica a necessidade de compreender as práticas zooterápicas no contexto da conservação da biodiversidade no Brasil (ALVES et al., 2007).
As práticas zooterápicas na área pesquisada seguem ainda uma vertente mística, as chamadas "curas mágicas" ou "simpatias", onde se percebeu uma tendência ao emprego de animais vivos, como acontece com o Cancão - Cyanocorax cyanopogon e o Jabuti - Chelonoidis carbonaria, animais creditados como capazes de curar doenças respiratórias através do rito simbólico de alimentálos com os restos de comida do enfermo. Doenças com causas sobrenaturais existem em diferentes culturas (RUBEL, 1977), logo, em função dos sistemas médicos tradicionais serem organizados como sistemas culturais, o uso das substâncias animais, além de uma perspectiva sociocultural, deve ser compreendido também dentro de uma perspectiva ecológica (COSTA-NETO, 2004).

Vale salientar, entretanto, que segundo relato dos próprios caçadores entrevistados, parte considerável do conhecimento e das práticas voltadas para tratamentos zooterápicos, etnoveterinários, cosméticos e místicos tem sido negligenciada atualmente, sobretudo por desinteresse dos mais jovens e acesso mais facilitado aos medicamentos alopáticos e aos tratamentos convencionais, o que explica os $\mathrm{VU}_{\mathrm{p}}$ acima de 0,3 para espécies com utilidade em tratamentos tradicionais de saúde humana e de animais de criação, a exemplo da Raposa - Cerdocyon thous $\left(\mathrm{VU}_{\mathrm{p}}=1,17\right)$; do Cancão - Cyanocorax cyanopogon $\left(\mathrm{VU}_{\mathrm{P}}=0,54\right)$; da Cascavel - Caudisona durisa $\left(\mathrm{VU}_{\mathrm{P}}=0,50\right)$; do Preá - Cavia aperea $\left(\mathrm{VU}_{\mathrm{P}}=0,42\right)$ e do Camaleão - Iguana iguana $\left(\mathrm{VU}_{\mathrm{P}}=0,38\right)$.

No presente trabalho notou-se que outro forte estimulador atual da caça de animais silvestres é o seu eventual potencial para atacar seres humanos ou criações de animais domésticos. Esses conflitos foram relatados pelos caçadores como recorrentes e, sobretudo, direcionados às serpentes, salientando todas as nove espécies de serpentes registradas apresentaram $\mathrm{VU}_{\mathrm{A}}$ mais elevado que $\mathrm{VU}_{\mathrm{P}}$, o que evidencia o elevado grau de risco a que essas espécies estão submetidas na região. Trinca e Ferrari (2006), em estudo a respeito da caça no assentamento rural Japuranã, município de Nova Bandeirantes, Mato Grosso, notaram que em 14,2\% dos casos de abate dos animais, estes eram caçados por predarem criações, caracterizando a caça de controle, ou por serem considerados perigosos aos animais domésticos 
e às pessoas. De modo geral, segundo Palmeira e Barrella (2007), embates do tipo frequentemente ocorrem quando animais selvagens danificam as safras, ferem ou matam animais domésticos, ameaçam ou matam pessoas.

$\mathrm{Na}$ região de estudo, percebe-se ainda que contradições e ambiguidades permeiam a conexão entre humanos e a fauna silvestre local, pois os animais tanto podem constituir-se em fonte de recursos quanto serem vistos como possibilidade de riscos, corroborando com Marques (2001). Normalmente, animais carnívoros silvestres não têm o habito de atacar criações domésticas, já que em ambientes que apresentam condições para a sua sobrevivência, esses animais evitam qualquer contato com o homem e suas criações, entretanto, devido à diminuição de suas presas naturais em virtude da caça predatória e/ou da fragmentação do habitat, os carnívoros podem atacar espécies domésticas (AZEVEDO; CONFORTI, 2002). É o que pode estar acontecendo na área pesquisada, uma vez que os entrevistados relataram que o número de ataques aumentou à medida que o desmatamento para agricultura expandiu-se e se intensificaram as práticas e técnicas de caça.

As principais técnicas de caça e captura mencionadas pelos entrevistados foram a caça com arma de fogo e cães, o uso de armadilhas e o facheado (técnica praticada à noite, que consiste no uso de fachos de luz para ofuscar os animais enquanto estes são coletados, e que é geralmente aplicada a pequenas aves). Outras técnicas de caça mencionadas pelos entrevistados foram a caça com estilingue, a técnica de espera (que consiste em aguardar o animal em pontos específicos, como árvores frutíferas e mananciais de água) e o arremedo (que usa apitos para imitar o canto das aves e assim atraí-las). Todas as técnicas descritas no presente trabalho já haviam sido registradas por Alves et al. (2009) no semiárido paraibano, o que sugere uma disseminação consistente dos conhecimentos e técnicas cinegéticas na região.

O ensino dos modos de captura dos animais, dos melhores períodos de caça e dos locais onde se encontrar cada espécie, bem como das variadas maneiras de se aproveitá-las, configura uma prática comum entre os habitantes da comunidade pesquisada, que executam também a caça como atividade de lazer, de forma costumeira e disseminada culturalmente de geração a geração. As atividades de caça nas regiões estudadas começam na infância quando pequenos animais são caçados como forma de "diversão" com uso de estilingues, ou capturadas em armadilhas e criadas como animais de estimação. Alves et al. (2009), que realizaram trabalho sobre as estratégias de caça usadas em Pocinhos, semiárido paraibano, verificaram também o uso, inclusive por crianças, das mesmas técnicas de caça do presente estudo. Trinca e Ferrari (2006), em trabalho acerca da caça em um assentamento rural na Amazônia mato-grossense, também relatam algumas dessas técnicas de captura. Como a exploração da fauna silvestre no continente americano tradicionalmente se dá através da caça (MANDUJANO; RICO-GRAY, 1991; ZAPATA, 2001), as atividades cinegéticas aliadas às diversas modalidades de aproveitamento dos recursos animais têm sido reconhecidas como práticas tradicionais transmitidas de geração a geração (BARRERABASSOLS; TOLEDO, 2005; MARTÍNEZ, 2006), o que demonstra a importância de registros do tipo como ferramenta para estudos mais aprofundados acerca da dinâmica de aproveitamento da fauna e seu histórico de conservação.

Conclui-se, assim, que na comunidade estudada há clara evidência de que as interações de uso existentes entre humanos e fauna constituem uma das mais significativas formas de aproveitamento dos recursos naturais da região, sobretudo como importante fonte de proteína animal. Além disso, a situação econômica também mostrou-se forte influenciadora da exploração cinegética na área.

De um modo menos significativo, a utilização tradicional de sobprodutos de interesse medicinal, veterinário, estético e místico também responde por parte da demanda exploratória da fauna na região.

A análise aprofundada de características históricas, socioculturais e econômicas locais será fundamental no momento de tentar avaliar as implicações das modalidades de relação que os seres humanos vivenciam com os animais da região, bem como será também para a elaboração, por meio de órgãos competentes, de políticas públicas de manutenção da cultura e do ambiente na área. 


\section{Agradecimentos}

Os autores gostariam de agradecer pela colaboração do Prof. Dr Rômulo Romeu da Nóbrega Alves (UEPB); à Universidade Federal de Campina Grande (UFCG) / Coordenação de Aperfeiçoamento de Pessoal de Nível Superior (CAPES) pela bolsa de pós-graduação concedida ao primeiro autor. Agradecimentos especiais a todos os entrevistados, que gentilmente compartilharam conosco seus conhecimentos.

\section{Referências}

AlBuQuerQue, U. P.; LUCENA, R. F. P. (Org.). Métodos e técnicas na pesquisa etnobotânica. Recife: Livro Rápido, NUPEEA, 2004. 323 p.

ALMEIDA, S. M; FRANCHIN, A. G.; MARÇAL JÚNIOR, O. Estudo etnoornitológico no Distrito Rural de Florestina, município de Araguari, Região do Triângulo Mineiro, Minas Gerais. Sitientibus: Série Ciências Biológicas, Feira de Santana, v. 6, p. 26-36, 2006.

ALVES, R. R. N. Fauna used in popular medicine in Northeast Brazil. Journal of Ethnobiology and Ethnomedicine, London, v. 5, n. 1, p. 1-11, 2009.

ALVES, R. R. N.; BARBOSA, J. A. A.; SANTOS, S. L. D. X.; SOUTO, W. M. S.; BARBOZA, R. R. D. Animal-based remedies as complementary medicines in the semi-arid region of northeastern Brazil. Evidence-Based Complementary and Alternative Medicine, New York, 2011. doi:10.1093/ecam/nep134.

ALVES, R. R. N.; MENDONÇA, L. E. T.; CONFESSOR, M. V. A.; VIEIRA, W. L. S.; LOPEZ, L. C. S. Hunting strategies used in the semi-arid region of northeastern Brazil. Journal of Ethnobiology and Ethnomedicine, London, v. 5, n. 12, p. 1-16, 2009.

ALVES, R. R. N.; ROSA, I. L. From cnidarians to mammals: The use of animals as remedies in fishing communities in NE Brazil. Journal of Ethnopharmacology, Shannon, v. 107, p. 259-276, 2006.

ALVES, R. R. N.; ROSA, I. L. Zootherapeutic practices among fishing communities in North and Northeast Brazil: a comparison. Journal of Ethnopharmacology, Shannon, v. 111, p. 82-103, 2007a.

ALVES, R. R. N.; ROSA, I. L. Zootherapy goes to tawn: the use of animal-based remedies in urban areas of NE and N Brazil. Journal of Ethnopharmacology, Shannon, v. 113, p. 541-555, $2007 \mathrm{~b}$.

ALVES, R. R. N.; ROSA, I. L.; SANTANA G. G. The role of animal-derived remedies as complementary medicine in Brazil. BioScience, Berkeley, v. 57, p. 949-955, 2007.

AYRES, J. M.; AYRES C. Aspectos da caça no alto Rio Aripuanã. Acta Amazonica, Manaus, v. 9, n. 2, p. 287-298, 1979.

AZEVEDO, F. C. C.; CONFORTI, V. A. Fatores predisponentes à predação. In: LEITE-PITMAN, M. R. P.; OLIVEIRA, T. G.; PAULA, R. C.; INDRUSIAK, C. (Ed.). Manual de identificação, prevenção e controle de predação por carnívoros. Brasília, Edições IBAMA, 2002. p. 27-28.

BARBOSA, J. A. A. Breves tópicos acerca da Lei De Diretrizes e Bases da Educação Nacional e a necessidade urgente de seu cumprimento exato em regiões do semiárido paraibano. Revista Âmbito Jurídico, Rio Grande, v. XIV, n. 90, p. 1-9, 2011.

BARBosA, J. A. A.; LEITE, D. A. B.; AGUIAR, J. O. Conflitos decorrentes da caça no semiárido nordestino: um estudo de caso no município de Fagundes, PB. Revista Âmbito Jurídico, Rio Grande, v. XIV, n. 102, p. 1-10, 2012.

BARBOSA, J. A. A.; NOBREGA, V. A.; Alves, R. R. N. Aspectos da Caça e Comércio Ilegal da Avifauna Silvestre por Populações Tradicionais do Semi-árido Paraibano. Revista de Biologia e Ciências da Terra, Campina Grande, v. 10, n. 2, p. 39-49, 2010.

BARBOSA, J. A. A.; NOBREGA, V. A.; ALVES, R. R. N. Hunting practices in the semiarid region of Brazil. Indian Journal of Traditional Knowledge, New Delhi, v. 10, n. 3, p. 486-490, 2011. BARRERA-BASSOLS, N.; TOLEDO, V. Ethnoecology of the Yucatec maya: Symbolism, knowledge and management of natural resources. Journal of Latin American Geography, Austin, v. 4, n. 1, p. 9-41, 2005.

BEGOSSI, A.; BRAGA, F. M. S. Food taboos and folk medicine among fishermen from the Tocantins River. Amazoniana, Plön, v. 12, p. 101-118, 1992.

BODMER, R.; PEZO, E. Rural development and sustainable wildlife use in Perú. Conservation Biology, San Francisco, v. 15, n. 4, p. 1163-1170, 2001.

CADIMA, C. I.; MARÇAL JÚNIOR, O. Notas sobre Etnoornitologia na comunidade do Distrito Rural de Miraporanga, Uberlândia, MG. Bioscience Journal, Uberlândia, v. 20, n. 1, p. 81-91, 2004.

CALOURO, A. M. Caça de subsistência: sustentabilidade e padrões de uso entre seringueiros ribeirinhos e não ribeirinhos do estado do Acre. 1995. 59 f. Dissertação (Mestrado em ecologia) Universidade de Brasília, Brasília. 1995.

CHIZOTTI, A. Pesquisa em ciências humanas e sociais. São Paulo: Cortez Editora, 2000. 27 p.

COSTA-NETO, E. M. Healing with animals in Feira de Santana City, Bahia, Brazil. Journal of Ethnopharmacology, Shannon, v. 65, p. 225-230, 1999.

COSTA-NETO, E. M. Implications and applications of folk zootherapy in the State of Bahia, Northeastern Brazil. Sustainable Development, West Sussex, v. 12, p. 161-174, 2004.

CPRM - SERVIÇO GEOLÓGICO DO BRASIL. Diagnóstico do município de Fagundes, estado da Paraíba. Recife: CPRM/ PRODEEM, 2005.

EMÍDIO-SILVA, C. A caça de subsistência praticada pelos índios Parakanã (sudeste do Pará): características e sustentabilidade. 1998. 69 f. Dissertação (Mestrado em Ciências Biológicas) Museu Paraense Emílio Goeldi e Empresa Brasileira de Pesquisa Agropecuária, Belém. 1998.

GIOVANINI, D. 1ำ Relatório nacional sobre o tráfico de fauna silvestre. Brasília: Rede Nacional de Combate ao Tráfico de Animais - RENCTAS, 2002. 108 p.

GUAdAGNin, D. L.; PERELlO, L. F. C.; MENEGHETI, J. O. A situação atual da caça de lazer e manejo de áreas úmidas no Rio Grande do Sul. Neotropical Biology and Conservation, São Leopoldo, v. 2, n. 2, p. 63-70, 2007.

HANAZAKI, N.; ALVES, R. R. N.; BEGOSSI, A. Hunting and use of terrestrial fauna used by Caiçaras from the Atlantic Forest coast (Brazil). Journal of Ethnobiology and Ethnomedicine, London, v. 5, n. 36, p. 1-8, 2009.

HUNTINGTON, H. P. Using traditional ecological knowledge in science: methods and applications. Ecological Applications, Ithaca, v. 10, n. 5, p. 1270-1274, 2000. 
IBAMA. Lista das espécies da fauna brasileira ameaçadas de extinção. Anexo à Instrução Normativa $\mathrm{n}^{\circ} \mathrm{o}$ 3. Brasília: Ministério do Meio Ambiente, 2003.

IBGE - INSTITUTO BRASILEIRO DE GEOGRAFIA E ESTATÍSTICA. Censo Brasil. 2010. Disponível em: <http://www. ibge.gov.br/cidadesat>. Acesso em: 25 ago. 2011.

LEAL, I. R.; SILVA, J. M. C.; TABARELLI, M.; LACHER JR, T. E. Mudando o curso da conservação da biodiversidade na Caatinga do Nordeste do Brasil. Megadiversidade, Belo Horizonte, v. 1, n. 1, p. 139-146, 2005.

LUCENA, R. F. P.; MEDEIROS, P. M.; ARAÚJO, E. L.; ALVES, A. G. C.; ALBUQUERQUE, U. P. The ecological apparency hypothesis and the importance of useful plants: an assessment based on use-value. Journal of Environmental Management, Leiden, v. 96, n. 1, p. 106-115, 2012.

MANDUJANO, S.; RICO-GRAY, V. Hunting, use, and knowledge of the biology of the white-tailed deer (Odocoileus virginianus Hays) by the maya of central Yucatan. Journal of Ethnobiology, Denton, v. 11, n. 2, p. 175-183, 1991.

MARQUES, J. G. W. Aspectos ecológicos na etnoictiologia dos pescadores do Complexo Estuarino-Lagunar MundaúManguaba, Alagoas. 1991. 98 f. Tese (Doutorado em Ecologia) - Universidade Estadual de Campinas, Campinas. 1991.

MARQUES, J. G. W. Pescando pescadores: ciência e etnociência em uma perspectiva ecológica. 2. ed. São Paulo: NUPAUB/USP, 2001. 64 p.

MARQUES, J. G. W. O olhar (des)multiplicado. O papel do interdisciplinar e do qualitativo na pesquisa etnobiológica e etnoecológica. In: AMOROZO, M. C. M.; MINGG, L. C.; SILVA, S. M. P. (Ed.). Métodos de coleta e análise de dados em etnobiologia, etnoecologia e disciplinas correlatas. Rio Claro: UNESP/CNPq, 2002. p. 31-46.

MARTÍNEZ, P. N. L. Aprovechamiento de fauna silvestre en una comunidad aledaña a la Reserva de la Biosfera Los Petenes, Campeche. 2006. 86 f. Dissertação (Mestrado em Ecologia Humana) - Centro de Investigación y de Estudios Avanzados del Instituto Politécnico Nacional, Mérida, Yucatán. 2006.

MARTINS, E. S. A caça de subsistência de extrativistas na Amazônia: sustentabilidade, biodiversidade e extinção de espécies. 1993. 117 f. Dissertação (Mestrado em Ecologia) - Universidade de Brasília, Brasília. 1993.

MELLO, L. G. Antropologia cultural. Rio de Janeiro: Editora Vozes, 1995. $51 \mathrm{p}$.

MENDONÇA, L. E. T.; SOUTO, C. M.; ANDRELINO, L. L.; SOUTO, W. M. S.; VIEIRA, W. L. S.; ALVES, R. R. N. Conflitos entre pessoas e animais silvestres no semiárido paraibano e suas implicações para conservação. Sitientibus Série Ciências Biológicas, Feira de Santana, v. 11, p. 185-199, 2012.

MILNER-GULLAND, E.; BENNETT, E.; GROUP, S. A. M. W. M. Wild meat: the bigger picture. Trends in Ecology and Evolution, Cambridge, v. 18, n. 7, p. 351-357, 2003.

MMA - MINISTÉRIO DO MEIO AMBIENTE. Avaliação das ações prioritárias para a conservação da biodiversidade da Caatinga. Brasília: UFPE/ FAD/CI do Brasil, Fundação Biodiversitas, EMBRAPA-Semi-Árido, MMA/SBF, 2002. 36 p.

MMA - MINISTÉRIO DO MEIO AMBIENTE. Status of the national biodiversity strategy advances. Meeting for identification of issues on biodiversity for cooperation and interchanging among South American countries. Brasília: MMA, 2003. 28 p.
MMA - MINISTÉRIO DO MEIO AMBIENTE. Lista nacional das espécies da fauna e da flora ameaçadas de extinção. Brasília: MMA, 2009.

MOURA, P. B. P.; MARQUE, J. G. W. Zooterapia popular na Chapada Diamantina: uma medicina incidental? Ciências \& Saúde Coletiva, Rio de Janeiro, v. 13, n. 2, p. 2179-2188, 2008.

NOBREGA, V. A.; BARBOSA, J. A. A.; ALVES, R. R. N. Utilização de aves silvestres por moradores do município de Fagundes, semiárido paraibano: uma abordagem etno-ornitológica. Sitientibus Série Ciências Biológicas, Feira de Santana, v. 11, n. 2, p. 165-175, 2011.

OVERAL, W. L. Introduction to ethnozoology: what it is or could be. In: POSEY, D. A.; OVERAL, W. L. (Org.). Ethnobiology: implications and applications. Belém: MPEG, 1990. p. 127-129.

PALMEIRA, F. B. L.; BARRELLA, W. Conflitos causados pela predação de rebanhos domésticos por felinos em comunidades quilombolas na Mata Atlântica. Biota Neotropica, Campinas, v. 7 n. 1, p. 119-128, 2007.

PEREIRA, G. A.; BRITO, M. T. Diversidade de aves silvestres comercializadas nas feiras livres da Região Metropolitana de Recife, Pernambuco. Atualidades Ornitológicas, Ivaiporã, n. 126, p. 14. 2005.

PEREIRA, J. P. R.; SCHIAVETTI, A. Conhecimentos e usos da fauna cinegética pelos caçadores indígenas "Tupinambá de Olivença" (Bahia). Biota Neotropica, Campinas, v. 10, n. 1, p. 175-183, 2010.

POSEY, D. Indigenous ecological knowledge and development of the Amazon. In: MORAN, E. F. (Ed.). The dilemma of Amazonian development. Boulder: Westview, 1983. p. 225-257.

RIBEIRO, L. B.; SILVA, M. G. O comércio ilegal põe em risco a diversidade das aves no Brasil. Ciencia e Cultura, São Paulo, v. 59, n. 4, 2007.

ROCHA, M. S. P.; CAVALCANTI, P. C. M.; SANTOS, R. L.; ALVES, R. R. N. Aspectos da comercialização ilegal de aves nas feiras livres de Campina Grande, Paraíba, Brasil. Revista de Biologia e Ciências da Terra, Campina Grande, v. 6, n. 2, p. 204-221, 2006.

ROSSATO, S. C.; LEITÃO-FILHO, H. F. E.; BEGOSSI, A. Ethnobotany of caiçaras of the Atlantic Forest coast (Brazil). Economic Botany, New York, v. 53, p. 387-395, 1999.

ROUBIK, D. W. Pollination of cultivated plants in the tropics. Rome: FAO Agricultural Services Bulletin, Smithsonain Tropical Research Institute, 1995. 118 p.

RUBEL, A. J. "The epidemiology of a folk illness: susto in Hispanic America." In: DAVID, L. (Ed.). Culture, disease, and healing: studies in medical anthropology. New York: Macmillan Publishing Co., 1977. p. 119-128.

SMITH, N. J. H. Utilization of game along Brazil's transamazon highway. Acta Amazonic, Manaus, v. 6, n. 4, p. 455-466, 1976.

TRINCA, C. T.; FERRARI, S. F. Caça em assentamento rural na amazônia matogrossense. In: JACOBI, P.; FERREIRA, L. C. (Org.). Diálogos em ambiente e sociedade no Brasil. Indaiatuba: ANPPAS, Annablume, 2006. p. 155-167.

VANNUCCI-NETO, R. Aves silvestres em cativeiro: considerações gerais. Tráfico de Aves. O Curumim, São Paulo, n. 95, p. 4-5. 2000.

ZAPATA, G. Sustentabilidad de la cacería de subsistencia: el caso d cuatro comunidades Quichuas en la Amazonia Nororienta Ecuatoriana. Mastozoología Neotropical, Tucumán v. 8, n. 1, p. 59-66, 2001. 
ANEXO I: Espécies da fauna cinegética abatidas e/ou usadas na região; categorias de uso/interação e indicadores de valor de uso atual (VU A $_{\text {) }}$ potencial $\left(\mathrm{VU}_{\mathrm{p}}\right)$. Legenda: Zooterapia (A); Etnoveterinária (B); Uso cosmético (C); Uso/Interação místico-religiosa (D); Criação/ Domesticação (E), Comércio (F); Uso/Interação ornamental (G); Alimentação (H); Lazer/Recreação (I); Caça de controle (J).

\begin{tabular}{|c|c|c|c|c|c|c|c|c|c|c|c|c|c|}
\hline Grupo/Família/Nome Local/Identificação & A & B & $\mathbf{C}$ & D & $\mathbf{E}$ & $\mathbf{F}$ & G & $\mathbf{H}$ & $\mathbf{I}$ & $\mathbf{J}$ & Total & $\mathbf{V} \mathbf{U}_{\mathrm{A}}$ & $\mathbf{V U}_{\mathbf{P}}$ \\
\hline \multicolumn{14}{|l|}{ Anfíbios } \\
\hline \multicolumn{14}{|l|}{ Bufonidae } \\
\hline Sapo/Cururu - Rhinella jimi (Stevaux, 2002) & 5 & 3 & & & & & & & & 1 & 9 & 0,13 & 0,25 \\
\hline \multicolumn{14}{|l|}{ Leiuperidae } \\
\hline \multicolumn{14}{|l|}{ Leptodactylidae } \\
\hline Jia - Leptodactylus vastus A. Lutz, 1930 & 4 & & & & & & & 7 & & & 11 & 0,42 & 0,04 \\
\hline \multicolumn{14}{|l|}{ Répteis } \\
\hline \multicolumn{14}{|l|}{ Boidae } \\
\hline \multicolumn{13}{|l|}{ Chelidae } & 0,17 \\
\hline \multicolumn{13}{|l|}{ Colubridae } & 0,25 \\
\hline Cobra-cipó - Oxybelis aeneus (Wagler, 1824) & & & & & & & & & & 5 & 5 & 0,21 & 0,00 \\
\hline Cobra preta - Pseudoboa nigra (Dumeril, 1854) & & & & 13 & & & & & & 3 & 16 & 0,37 & 0,29 \\
\hline Cobra verde - Philodryas aestivus (Dumeril, 1854) & & & & & & & & & & 6 & 6 & 0,25 & 0,00 \\
\hline Corre campo - Philodryas nattereri (Steindachner, 1870) & & & & & & & & & & 5 & 5 & 0,21 & 0,00 \\
\hline Jararaquinha - Liophis poecilogyrus (Wied, 1825) & & & & & & & & & & 7 & 7 & 0,21 & 0,08 \\
\hline Papa-pinto - Spilotes pullatus (Linnaeus, 1758) & & & & & & & & & & 5 & 5 & 0,21 & 0,00 \\
\hline \multicolumn{14}{|l|}{ Crotalidae } \\
\hline \multicolumn{14}{|l|}{ Elapidae } \\
\hline \multicolumn{14}{|l|}{ Iguanidae } \\
\hline \multicolumn{14}{|l|}{ Teiidae } \\
\hline $\begin{array}{l}\text { Teju - Tupinambis merianae (Duméril \& Bibron, 1839) } \\
\text { Testudinidae }\end{array}$ & 17 & 11 & & & & 2 & & 9 & & 7 & 46 & 1,75 & 0,17 \\
\hline $\begin{array}{l}\text { Jabuti - Chelonoidis carbonaria (Spix, 1824) } \\
\text { Troniduridae }\end{array}$ & 3 & & & 2 & 5 & & & & & & 10 & 0,21 & 0,21 \\
\hline Lagartixa - Tropidurus histipus (Spix, 1825) & 4 & & & & \multicolumn{9}{|c|}{ Tropiduridae } \\
\hline \multicolumn{14}{|l|}{ Aves } \\
\hline$\overline{\text { Accipitridae }}$ & & & & & & & & & & & & & \\
\hline Gavião - Rupornis magnirostris (Gmelin, 1788) & & & & & & & & & & 4 & 4 & 0,00 & 0,17 \\
\hline Peneira - Elanus leucurus (Vieillot, 1818) & & & & & & & & & & 4 & 4 & 0,00 & 0,17 \\
\hline Caprimulgidae & & & & & & & & & & & & & \\
\hline Bacurau - Nyctidromus albicollis (Gmelin, 1789) ${ }^{\mathrm{LR}}$ & & & & & 2 & 4 & & & & & 6 & 0,00 & 0,25 \\
\hline Cardinalidae & & & & & & & & & & & & & \\
\hline Azulão - Passerina brissonii (Lichtenstein, 1823) & & & & & 3 & 5 & & & 2 & & 10 & 0,42 & 0,00 \\
\hline Trinca ferro - Saltator similis d'Orbigny \& Lafresnaye, 1837 & & & & & 2 & 1 & & & 1 & & 4 & 0,13 & 0,04 \\
\hline Cathartidae & & & & & & & & & & & & & \\
\hline Urubu-Coragyps atratus (Bechstein, 1793) & 3 & & & 1 & & & & & & & 4 & 0,00 & 0,17 \\
\hline $\begin{array}{l}\text { Charadriidae } \\
\text { Tetéu - Vanellus chilensis (Molina 1782) LC }\end{array}$ & & & & & & & & & & & & & \\
\hline $\begin{array}{l}\text { Tetéu - Vanellus chilensis (Molina, 1782) LC } \\
\text { Columbidae }\end{array}$ & & & & 2 & & & & & & & 2 & 0,00 & 0,08 \\
\hline Asa branca - Columba picazuro Temminck, $1813^{\text {LC }}$ & 4 & & & 5 & & & & & & & 9 & 0,00 & 0,38 \\
\hline Burguesa branca - Streptopelia sp. & & & & & 2 & 5 & & 7 & & & 14 & 0,58 & 0,00 \\
\hline Burguesa marrom - Streptopelia decaocto Frivaldszky, $1838^{\text {LC }}$ & & & & & 2 & 5 & & 7 & & & 14 & 0,58 & 0,00 \\
\hline Juruti - Leptotila rufaxilla (Richard \& Bernard, 1792) ${ }^{\mathrm{LC}}$ & 1 & & & & & & & 1 & & & 2 & 0,00 & 0,08 \\
\hline Ribaçã - Zenaida auriculata (Des Murs, 1847) & & & & & & 10 & & 19 & 3 & & 32 & 1,33 & 0,00 \\
\hline Rolinha-Columbina sp. & & & & & & 10 & & 19 & 3 & & 32 & 1,33 & 0,00 \\
\hline Rolinha cambuta - Columbina minuta (Linnaeus, 1766) & & & & & & & & 11 & 2 & & 13 & 0,54 & 0,00 \\
\hline Rolinha prateada- Columbina sp. & & & & & & & & 9 & 2 & & 11 & 0,46 & 0,00 \\
\hline Corvidae & & & & & & & & & & & & & \\
\hline Cancão - Cyanocorax cyanopogon (Wied, 1821) & 6 & & & 8 & 8 & & & & & & 22 & 0,38 & 0,54 \\
\hline Emberizidae & & & & & & & & & & & & & \\
\hline Bigode - Sporophila lineola (Linnaeus, 1758) & & & & & 3 & 3 & & & & & 6 & 0,25 & 0,00 \\
\hline Caboclinho - Sporophila bouvreuil (Miller, 1776) & & & & & 3 & 2 & & & & & 5 & 0,21 & 0,00 \\
\hline Canário - Sicalis flaveola (Linnaeus 1766) & & & & & 2 & 2 & 1 & & 2 & & 7 & 0,29 & 0,00 \\
\hline Chorão - Sporophila leucoptera (Vieillot 1817) & & & & & 1 & 2 & & & & & 3 & 0,13 & 0,00 \\
\hline Curió - Oryzoborus angolensis Linnaeus, $1766^{\mathrm{LC}}$ & & & & & 1 & 1 & & & & & 2 & 0,08 & 0,00 \\
\hline Galo de campina - Paroaria dominicana (Linnaeus, 1758) & & & & & 5 & 9 & 2 & & 4 & & 20 & 0,83 & 0,00 \\
\hline
\end{tabular}




\begin{tabular}{|c|c|c|c|c|c|c|c|c|c|c|c|c|c|}
\hline Grupo/Família/Nome Local/Identificação & $\mathbf{A}$ & B & $\mathbf{C}$ & D & $\mathbf{E}$ & $\mathbf{F}$ & $\mathbf{G}$ & $\mathbf{H}$ & $\mathbf{I}$ & $\mathbf{J}$ & Total & $\mathbf{V} \mathbf{U}_{\mathbf{A}}$ & $\mathbf{V U}_{\mathrm{P}}$ \\
\hline Gaturão - Sicalis luteola (Sparrman, 1789) & & & & & 4 & 2 & & & 1 & & 7 & 0,29 & 0,00 \\
\hline Golado - Sporophila albogularis (Spix, 1825) & & & & & 7 & 9 & & & 2 & & 18 & 0,75 & 0,00 \\
\hline Papa-capim - Sporophila nigricollis (Vieillot 1823) & & & & & 2 & 1 & & & 1 & & 4 & 0,17 & 0,00 \\
\hline Salta caminho - Tiaris fuliginosus (Wied, 1830) & & & & & 1 & & & & 1 & & 2 & 0,08 & 0,00 \\
\hline Tico-tico - Zonotrichia capensis (Müller, 1776) & & & & & 2 & 1 & & & 1 & & 4 & 0,17 & 0,00 \\
\hline Tiziu - Volatinia jacarina (Linnaeus, 1766) & & & & & 3 & 2 & & & 1 & & 6 & 0,25 & 0,00 \\
\hline \multicolumn{14}{|l|}{ Estrildidae } \\
\hline Bico de lápis - Estrilda astrild Linnaeus, 1758 & & & & & 2 & 2 & & & 1 & & 5 & 0,21 & 0,00 \\
\hline \multicolumn{14}{|l|}{ Falconidae } \\
\hline Acauã - Herpetotheres cachinnans (Linnaeus, 1758) LR/II & 3 & & & 7 & & & & & & & 10 & 0,13 & 0,29 \\
\hline Carcará - Caracara plancus (Miller, 1777) & & & & & & & & & & 5 & 5 & 0,21 & 0,00 \\
\hline \multicolumn{14}{|l|}{ Fringillidae } \\
\hline Canário belga - Serinus canaria (Linnaeus, 1758) & & & & & 2 & 1 & & & 1 & & 4 & 0,17 & 0,00 \\
\hline Corda negra - Agelaius ruficapillus Vieillot, $1819^{\text {LC }}$ & & & & & 4 & 7 & & & 2 & & 13 & 0,54 & 0,00 \\
\hline Pinta silva - Carduelis yarrellii (Audubon, 1839) & & & & & 2 & 1 & & & & & 3 & 0,13 & 0,00 \\
\hline Vem-vem - Euphonia chlorotica (Linnaeus, 1776) & & & & 2 & 1 & 1 & & & & & 4 & 0,08 & 0,08 \\
\hline \multicolumn{14}{|l|}{ Furnariidae } \\
\hline Casaca de couro - Furnarius leucopus Swainson, 1837 & & & & & 3 & 3 & & & 2 & & 8 & 0,33 & 0,00 \\
\hline \multicolumn{14}{|l|}{ Icteridae } \\
\hline Concriz - Icterus jamacaii (Gmelin, 1788) & & & & & 3 & 6 & & & 1 & & 10 & 0,42 & 0,00 \\
\hline Craúna - Gnorimopsar chopi (Vieillot, 1819) ${ }^{\mathrm{LC}}$ & & & & & 2 & 2 & & & 1 & & 5 & 0,21 & 0,00 \\
\hline Xexeu-de-bananeira - Icterus cayanensis (Linnaeus, 1766) ${ }^{\mathrm{LC}}$ & & & & & 1 & & & & & & 1 & 0,04 & 0,00 \\
\hline \multicolumn{14}{|l|}{ Psitacidae } \\
\hline Papagaio - Amazona aestiva (Linnaeus, 1758) & 2 & & 2 & & 3 & 1 & 1 & & 1 & & 10 & 0,21 & 0,21 \\
\hline \multicolumn{14}{|l|}{ Rallidae } \\
\hline Galinha d'água - Gallinula chloropus, (Linnaeus, 1758) & & & & & & 5 & & 12 & & & 17 & 0,58 & 0,13 \\
\hline \multicolumn{14}{|l|}{ Thraupidae } \\
\hline Sanhaçu - Thraupis sayaca (Linnaeus, 1766) & & & & & 2 & & & & & & 2 & 0,08 & 0,00 \\
\hline \multicolumn{14}{|l|}{ Tinamidae } \\
\hline Lambu - Crypturellus parvirostris (Wagler, 1827) & & & & & & 4 & & 17 & 2 & & 23 & 0,96 & 0,00 \\
\hline Lambu do pé roxo - Crypturellus tataupa (Temminck, 1815) & & & & & & 4 & & 14 & 2 & & 20 & 0,83 & 0,00 \\
\hline \multicolumn{14}{|l|}{ Trochilidae } \\
\hline Beija-flor - Amazilia fimbriata (Gmelin, 1788) ${ }^{\mathrm{LC}}$ & 1 & & & & & & 1 & & 1 & & 3 & 0,08 & 0,04 \\
\hline \multicolumn{14}{|l|}{ Turdidae } \\
\hline Sabiá - Turdus rufiventris (Vieillot, 1818) & & & & & 5 & 3 & 1 & & & & 9 & 0,38 & 0,00 \\
\hline Tyrannidae & & & & & & & & & & & & & \\
\hline Bem-te-vi - Pitangus sulphuratus (Linnaeus, 1766) ${ }^{\mathrm{LC}}$ & & & & 2 & & & & & & & 2 & 0,00 & 0,08 \\
\hline Lavandeira - Fluvicola nengeta (Linnaeus, 1766) & 4 & & & 3 & & & & & & & 7 & 0,21 & 0,08 \\
\hline Maria fita - Empidonax traillii (Audubon, 1828) & & & & & 4 & 2 & & & & & 6 & 0,25 & 0,00 \\
\hline Tytonidae & & & & & & & & & & & & & \\
\hline Coruja - Tyto alba (Scopoli, 1769) ${ }^{\mathrm{LC}}$ & & & & 5 & & & & & & & 5 & 0,21 & 0,00 \\
\hline Mamíferos & & & & & & & & & & & & & \\
\hline Canidae & & & & & & & & & & & & & \\
\hline Raposa - Cerdocyon thous (Linnaeus, 1766) & 13 & 8 & & 6 & & & 3 & & & 5 & 35 & 0,29 & 1,17 \\
\hline Caviidae & & & & & & & & & & & & & \\
\hline Mocó - Kerodon rupestris (Wied-Neuwied, 1820) LC & 4 & & & & & 5 & & 14 & 7 & & 30 & 0,96 & 0,29 \\
\hline Preá - Cavia aperea Erxleben $1777^{\text {LC }}$ & 6 & & 2 & & & 5 & & 16 & 7 & & 36 & 1,08 & 0,42 \\
\hline Cebidae & & & & & & & & & & & & & \\
\hline Sagüi - Callithrix sp. & & & & & 3 & & & & & & 3 & 0,13 & 0,00 \\
\hline Dasypodidae & & & & & & & & & & & & & \\
\hline Peba - Euphractus sexcinctus (Linnaeus, 1758) ${ }^{\text {LC }}$ & & & & & 11 & 7 & & 18 & 6 & & 42 & 1,63 & 0,13 \\
\hline Tatu - Dasypus novemcinctus (Linnaeus, 1758) vu & 3 & & & & 11 & 7 & & 18 & 6 & & 45 & 1,63 & 0,25 \\
\hline Didelphidae & & & & & & & & & & & & & \\
\hline Timbu - Didelphis albiventris (Lund, 1840) ${ }^{\mathrm{LC}}$ & 1 & & & & & & & 4 & 1 & 7 & 13 & 0,50 & 0,04 \\
\hline Felidae & & & & & & & & & & & & & \\
\hline Gato Maracajá - Leopardus wiedii ( Schinz, 1821) ${ }^{\mathrm{LC}}$ & & & & & & & 9 & & 5 & & 14 & 0,00 & 0,58 \\
\hline Molossidae & & & & & & & & & & & & & \\
\hline Morcego - Molossus molossus (Pallas, 1766) ${ }^{\mathrm{LC}}$ & 3 & & & & & & & & & 1 & 4 & 0,04 & 0,13 \\
\hline Mustelidae & & & & & & & & & & & & & \\
\hline Furão - Mustela putorius furo Linnaeus, 1758 & & & & & & & & & & 4 & 4 & 0,17 & 0,00 \\
\hline Tacaca - Conepatus semistriatus (Boddaert, 1785) ${ }^{\mathrm{LC}}$ & 2 & & & & & & & 4 & 2 & & 8 & 0,25 & 0,08 \\
\hline
\end{tabular}

